\title{
Anomalias da raiz - dilaceração e raiz supranumerária: revisão integrativa da literatura
}

\author{
Root anomalies - tearing and supernumerary root: integrative literature review \\ Anomalías de la raíz - raíz desgarrada y supernumeraria: revisión integrativa de la literatura
}

Paulo Fernando Lauria Fonseca ORCID: https://orcid.org/0000-0002-9446-2454 Centro Universitário do Estado do Pará, Brasil

E-mail: paulolauria@outlook.com

Andrezza Ozela de Vilhena

ORCID: https://orcid.org/0000-0002-2162-1311

Universidade do Estado do Pará, Brasil E-mail: aozelav@gmail.com

Bruna Renata Farias dos Santos ORCID: https://orcid.org/0000-0003-0228-8549

Hospital de Clínicas Gaspar Vianna, Brasil

E-mail: Santos.brf123@gmail.com

Danielle Freire Goncalves

ORCID: https://orcid.org/0000-0002-2469-1876 Universidade do Estado do Pará, Brasil

E-mail: adaniellefreire@gmail.com

Patricia Danielle Lima de Lima

ORCID: https://orcid.org/0000-0002-1068-2813 Universidade do Estado do Pará, Brasil E-mail: patricia.lima@uepa.br

Flavia Nunes Vieira

ORCID: https://orcid.org/0000-0002-1538-9130 Fundação Santa Casa de Misericórdia do Pará, Brasil E-mail: flaviavieira99@gmail.com

Marcia Helena Machado Nascimento

ORCID: https://orcid.org/0000-0003-1573-8991 Universidade do Estado do Pará, Brasil

E-mail: marcia.nascimento@uepa.br

Yanka Macapuna Fontel

ORCID: https://orcid.org/0000-0003-3150-6402 Universidade do Estado do Pará, Brasil

E-mail: yanka_fontel@hotmail.com

Rayssa da Silva Sousa

ORCID: https://orcid.org/0000-0003-4413-496X

Hospital de Clínica Gaspar Vianna, Brasil

E-mail: rayssasousaef_@hotmail.com

Juliana Rosario de Moraes

ORCID: https://orcid.org/0000-0002-1217-6471 Universidade do Estado do Pará, Brasil E-mail: enf.julianamoraes@hotmail.com

Gabriela Evelyn Rocha da Silva

ORCID: https://orcid.org/0000-0002-2488-9196

Hospital das Clínicas Gaspar Vianna, Brasil

E-mail: gabyevelyn16@gmail.com

Thiago dos Santos Carvalho

ORCID: https://orcid.org/0000-0001-7804-655X Universidade do Estado do Pará, Brasil E-mail: thiagolaenf@gmail.com

Sílvia Renata Pereira dos Santos ORCID: https://orcid.org/0000-0002-6517-8251

Hospital de Clínicas Gaspar Vianna, Brasil E-mail: renatapereira_8@ hotmail.com

Lucas Ferreira de Oliveira

ORCID: https://orcid.org/0000-0002-6799-6055 Universidade do Estado do Pará, Brasil E-mail: lucasf29.1f@gmail.com 


\begin{abstract}
Resumo
Objetivo: realizar uma revisão integrativa da literatura que exponha resultados em bancos de dados científicos acerca das anomalias da raiz - dilaceração e raiz supranumerária; realizar a síntese das informações acerca das anomalias da raiz - dilaceração e raiz supranumerária obtidas na literatura; elencar a importância de tal conhecimento para o aprimoramento assistencial odontológico. Metodologia: Trata-se de um estudo exploratório e descritivo, de abordagem qualitativa do tipo Revisão integrativa da literatura (RIL). Com a utilização do Iramuteq para elencar as classes discursivas resultantes das análises dos artigos selecionados. o banco de dados escolhido foi a BVS (biblioteca virtual de saúde), diante disto foram utilizados os seguintes critérios de inclusão: textos disponíveis gratuitamente e na íntegra na base de dados; textos em português e inglês; textos publicados entre 2010 - 2020; texto que contemplassem a temática abordada. Resultados e discussão: foram selecionados 5 artigos a partir da busca de banco de dados dentre esses artigos 3 foram de 2012, um de 2015 e um de 2020, expondo a carência de artigos acerca da temática, em relação às classe discursivas elencadas pelo iramuteq, o resultado foi ) Tratamentos para anomalias de raiz e o sofrimento ao paciente; 2) Efeitos da posição e angulação dentária em dilaceração da raiz; 3) Radiografia como exame fundamental para tratamento de anomalia de raiz; 4) fatores que colaboram para as anomalias de raiz.

Palavras chaves: Anomalias de raiz; Odontologia; Tratamento.
\end{abstract}

\begin{abstract}
Objective: to carry out an integrative literature review that presents results in scientific scientific databases about root anomalies - laceration and supernumerary root; perform the synthesis of information about root anomalies - laceration and complete supernumerary root in the literature; to list the importance of such knowledge for the improvement of dental care. Methodology: This is an exploratory and descriptive study with a qualitative approach of the Integrative Literature Review (RIL) type. Using Iramuteq to list the discursive classes of the analysis of selected articles. the chosen database was the VHL (virtual health library), these were used the following inclusion criteria: texts available for free and in the database; texts in Portuguese and English; texts published between 2010 - 2020; text that contemplated the addressed theme. Results and discussion: 5 articles were selected from the database search among these articles 3 were from 2012, one from 2015 and one from 2020, exposing the lack of articles on the subject, in relation to the discursive classes listed by iramuteq, the result was) Treatments for root anomalies and patient suffering; 2) Effects of tooth position and angulation on root tear; 3) Radiography as a fundamental exam for the treatment of root anomalies; 4) factors that contribute to root anomalies.
\end{abstract}

Keywords: Root anomalies; Dentistry; Treatment.

\title{
Resumen
}

Objetivo: realizar una revisión integradora de la literatura que presente resultados en bases de datos científicas científicas sobre anomalías radiculares - laceración y raíz supernumeraria; realizar la síntesis de información sobre anomalías de la raíz: laceración y raíz supernumeraria completa en la literatura; enumerar la importancia de dicho conocimiento para la mejora del cuidado dental. Metodología: Se trata de un estudio exploratorio y descriptivo con enfoque cualitativo del tipo Revisión Integrativa de Literatura (RIL). Uso de Iramuteq para enumerar las clases discursivas del análisis de artículos seleccionados. La base de datos elegida fue la BVS (biblioteca virtual en salud), se utilizaron los siguientes criterios de inclusión: textos disponibles de forma gratuita y en la base de datos; textos en portugués e inglés; textos publicados entre 2010 - 2020; texto que contempló el tema abordado. Resultados y discusión: Se seleccionaron 5 artículos de la búsqueda en la base de datos entre estos artículos 3 fueron del 2012, uno del 2015 y uno del 2020, exponiendo la falta de artículos sobre el tema, en relación a las clases discursivas enumeradas por iramuteq, el resultado fue ) Tratamientos para anomalías radiculares y sufrimiento del paciente; 2) Efectos de la posición y la angulación de los dientes sobre el desgarro de la raíz; 3) La radiografía como examen fundamental para el tratamiento de anomalías radiculares; 4) factores que contribuyen a las anomalías de la raíz.

Palabras clave: Anomalías de la raíz; Odontología; Tratamiento.

\section{Introdução}

O presente artigo visa a realização de uma revisão da literatura que aborde produções científicas que colabore para estudos acerca das Anomalias da raiz - dilaceração e raiz supranumerária. Diante disso ressalta-se que a anatomia dentária é fundamental para que todo o dentista saiba observar os dentes e todas as estruturas orais circunvizinhas, para que, no momento em que se deparar com alterações clínicas e radiográficas que fogem às condições anatómicas normais, realize um diagnóstico com precisão, estabeleça um plano de tratamento adequado e faça o devido acompanhamento do doente. Para tal, os profissionais devem ter todo o conhecimento relacionado com o desenvolvimento, morfologia, função e identidade da dentição humana (Faria, 2003). Como afeta as raízes dentais é identificada apenas através de exames radiográficos, sendo na maioria das 16 vezes um achado identificado em uma imagem solicitada para outro fim, não relacionado a anomalia (Cral, 2016). 
A investigação das alterações e dos processos patológicos que afetam o complexo dentomaxilofacial destes pacientes constitui um capítulo importante dentro do estudo das lesões orais, uma vez que condições que acontecem na infância podem ter um impacto importante na saúde geral da criança e podem trazer repercussões para a vida adulta (Tommasi, 2014). Na literatura, são escassos os estudos que mostram a ocorrência de lesões orais e anomalias dentárias diagnosticadas em radiografias panorâmicas de pacientes, sobretudo no Brasil. Além disso, verifica-se que há uma grande variabilidade, a depender de aspectos relacionados à população de estudo, assim como dos critérios de diagnóstico utilizados (Lucas, 2019).

A dilaceração radicular é uma anomalia dentária de forma caracterizada por uma curvatura anormal na raiz em relação a coroa do dente, pode ser encontrada na literatura como acotovelamento dentário, fratura traumática intrafolicular, luxação traumática intrafolicular, desvio ou curva na relação linear da coroa de um dente com sua raiz (Dutra et.al, 2007). A incidência dessa anomalia é baixa, não há predileção de sexo e é muito raro ocorrer em mais de um elemento dentário principalmente da mesma arcada (Jafarzadeh,2007).

Ocorre uma angulação anormal da raiz e ou menos frequentemente, da coroa dentária. Pode comprometer a irrupção espontânea do dente, causando desde um atraso até a retenção intra-óssea. Os dentes mais acometidos são os caninos superiores, seguidos pelos segundos pré-molares inferiores e incisivos centrais superiores. A ausência clínica do dente permanente é um sinal importante no diagnóstico de dilaceração radicular, sendo o exame radiográfico essencial (Paula et.al, 2008).

Sua etiologia está associada a fatores traumáticos na dentição decídua, ou a fatores hereditários como desenvolvimento anormal da raiz devido à presença de dentes supranumerários, cistos ou tumores adjacentes, desenvolvimento ectópico do germe dentário, falta de espaço, anormalidade endócrina, doença óssea, lábio leporino e fenda palatina, erupção retardada generalizada, anomalia dental ou tecidual (Silva et.al, 2012).

Raiz supranumerária refere-se ao aumento do número de raízes dentárias comparado aos padrões anatômicos de normalidade. Esta anomalia é identificada através do exame radiográfico e, é melhor visualizada quando se apresenta divergente, visto que o tamanho, a posição e a sobreposição de estruturas anatômicas podem dificultar a visualização. A presença de espaços duplos do ligamento periodontal em um dos lados da raiz ou de espaço do ligamento periodontal atravessando as raízes podem ser indicativos da presença de uma raiz supranumerária (Cral, 2016).

Os dentes podem apresentar raízes acessórias ou supranumerárias. O desenvolvimento de uma raiz supranumerária pode estar relacionado a trauma, pressão ou doença metabólica que afeta a bainha epitelial de Hertwig. Raízes supranumerárias podem ser totalmente desenvolvidas em tamanho e forma ou pequenas e rudimentares (Theodorovicz,2012).

Pautados na justificativa de que é importante saber como ocorre a atuação do cirurgião-dentista em relação ao plano de tratamento adequado para se promover ações em prol da qualidade do atendimento ao usuário, pergunta-se: quais dados obtidos na literatura científica colaboram com informações acerca das anomalias da raiz - dilaceração e raiz supranumerária?

Para responder à questão enunciada, propõe-se como objetivo geral do estudo realizar uma revisão integrativa da literatura que exponha resultados em bancos de dados científicos acerca das anomalias da raiz - dilaceração e raiz supranumerária. Apresentando os seguintes objetivos específicos: realizar a síntese das informações acerca das anomalias da raiz - dilaceração e raiz supranumerária obtidas na literatura; elencar a importância de tal conhecimento para o aprimoramento assistencial odontológico.

\section{Metodologia}

Trata-se de um estudo exploratório e descritivo, de abordagem qualitativa do tipo Revisão integrativa da literatura (RIL). O estudo descritivo é comum no campo da educação, em que o foco é descrever de forma mais aprofundada o comportamento dos fenômenos/pessoas inseridas em uma realidade (Prodanov; Freitas, 2013). 
Optou-se pelo desenvolvimento da abordagem qualitativa, devido a abordagem qualitativa fundamentar-se nos princípios que os conhecimentos sobre os indivíduos só são possíveis com a descrição da experiência humana tal como ela é vivida e tal como ela é defendida por seus próprios autores (Santos, 2019). O que será possível por meio da realização da RIL.

Haja vista que o método de revisão integrativa permite a combinação de dados da literatura empírica e teórica que podem ser direcionados à definição de conceitos e identificação de lacunas nas áreas de estudos (Mattos, 2015). Além de a revisão integrativa determina o conhecimento atual sobre uma temática específica, já que foi conduzida de modo a identificar, analisar e sintetizar resultados de estudos independentes sobre o mesmo assunto, contribuindo, pois, para uma possível repercussão benéfica na qualidade dos cuidados prestados ao paciente permitindo assim a elaboração do estudo exploratórios descritivo (SANTOS, 2019).

Mattos (2015) determina seis etapas de produção de uma revisão da literatura, sendo elas: Estabelecimento da problemática, Estabelecimentos da fonte de dados e dos critérios para inclusão e exclusão de estudos, Definições das informações a serem extraídas dos artigos selecionados (Seleção dos dados), Avaliação dos estudos incluídos na revisão integrativa, Interpretação dos resultados, síntese do conhecimento (discussão das temáticas).

\section{Primeira etapa: Estabelecimento da problemática}

Para a elaboração desta primeira etapa, a presente RIL foi guiada pela questão formuladas a partir da técnica PICO, que tem a seguinte organização P - Pessoa ou Problema, I - Intervenção, C - Comparação, O - Resultado (Santos; Galvão et al., 2014). Para a construção da questão norteadora da revisão, foi utilizada a estratégia PICo adaptada em que P (pessoa/ problema): Raiz dentária, I (interesse): anomalias de raiz, Co (contexto): dilaceração e raiz supranumerária. Assim, questionou-se: Quais conteúdos sobre Anomalias da raiz - dilaceração e raiz supranumerária estão presentes na literatura para realização de uma RIL?

\section{Segunda etapa: Estabelecimentos da fonte de dados e dos critérios para inclusão e exclusão de estudos}

Nesta etapa o banco de dados escolhido foi a BVS (biblioteca virtual de saúde), diante disto foram utilizados os seguintes critérios de inclusão: textos disponíveis gratuitamente e na íntegra na base de dados; textos em português e inglês; textos publicados entre 2010 - 2020; texto que contemplassem a temática abordada.

Para isso foram usadas as combinações conforme Figura 1 na qual há presença do operador booleano and para aprimorar o refinamento das buscas.

Figura 1: Expõe os termos de busca e suas combinações nos bancos de dados.

\begin{tabular}{|l|l|l|l|l|l|l|}
\hline Descritor 1 & OB & Descritor 2 & OB & Descritor 3 & OB & Descritor 4 \\
\hline Raiz dentária & AND & anomalias & AND & assistência & AND & \\
\hline Raiz dentária & AND & Alterações & AND & tratamento & AND & \\
\hline Raiz dentária & AND & anomalias & AND & dilaceração & AND & supra numérica \\
\hline
\end{tabular}

Fonte: Autores (2021). 


\section{Terceira etapa: Definições das informações a serem extraídas dos artigos selecionados (Seleção dos dados)}

Nesta etapa foi utilizado um instrumento adaptado do formulário Ursi (2005). As informações extraídas foram: Título do artigo; título do periódico; número e nomes de autores; titulação dos autores; país e idioma da publicação; ano de publicação; instituição na qual o estudo foi realizado; tipo de publicação; característica metodológica do estudo; objetivo da pesquisa; característica das amostras; intervenções realizadas; resultados; conclusões da pesquisa.

\section{Quarta etapa: Avaliação dos estudos incluídos na revisão integrativa}

Esta etapa assemelhou-se à análise dos dados em uma pesquisa convencional, na qual houve o emprego do questionário supracitado na etapa anterior. Para garantir a validade da revisão, os estudos selecionados foram analisados detalhadamente. A princípio foi elaborada uma leitura flutuante dos artigos apenas dos títulos e resumos a fim de avaliar aproximação com a temática abordada; após suas seleções, foi realizada a leitura exaustiva, na qual os artigos foram lidos na íntegra.

\section{Quinta etapa: Interpretação dos resultados}

Foi utilizado o software IRAMUTEQ (Interface de $\mathrm{R}$ pour les Analyses Multidimensionnelles de Textes et de Questionnaires) que permite a partir da leitura do Corpus que é um texto codificado na linguagem apropriada para a análise dos resultados expressos pelos artigos selecionados, elencar as principais classes discursivas, a partir da análise hierárquica descendente (método de Reinert) em que os textos são classificados em função de seus respectivos vocabulários e o conjunto deles se divide pela frequência das formas reduzidas. A partir de matrizes que cruzam segmentos de textos e palavras (repetidos testes $\mathrm{X}^{2}$ ), aplica-se o método de CHD para obter uma classificação estável e definitiva (SOUZA et al., 2018). Formulando assim as classes discursivas que seriam tópicos abordados pelos artigos que enalteceram e trouxessem os resultados esperados pela RIL.

\section{Sexta etapa: síntese do conhecimento (discussão das temáticas)}

Após a elaboração das classes pela análise do IRAMUTEQ, elas serão discutidas, no intuito de expor as principais informações contidas na literatura acerca da Anomalias da raiz - dilaceração e raiz supranumerária.

\section{Resultados e Discussão}

Obteve-se como resultado da pesquisa a partir do estabelecimento das fontes de dados e dos critérios para inclusão e exclusão de estudos, 5 artigos que contemplavam os quesitos supracitados a partir da busca nos bancos de dados utilizando os descritores conectados pelo operador booleano AND, obteve-se os resultados expressados pela Figura 2 a qual expõe o diagrama de busca e seleção de artigos na BVS, para facilitar o processo de agrupamento dos dados obtidos, a figura subdivide-se em 2 esquemas (A e B) referentes ao resultado de buscas correspondentes a cada combinação de descritores de busca interligados por meio do Operador Booleano (OB) AND efetivadas durante a pesquisa. 
Figura 2: Dendograma de busca de artigos em banco de dados.

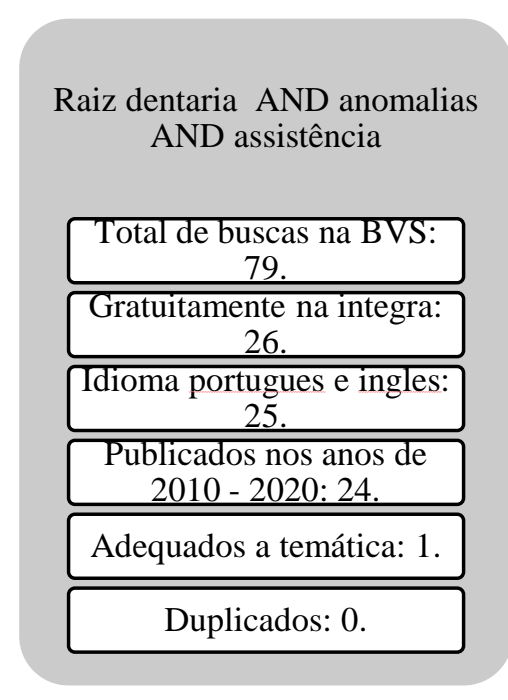

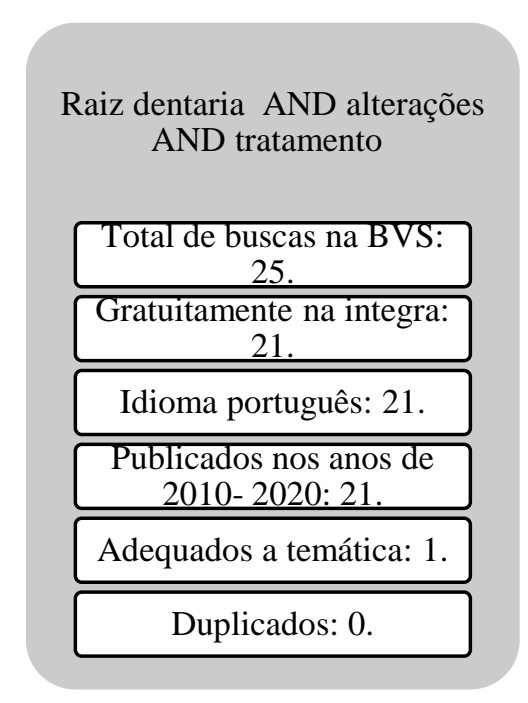

Fonte: Autores (2021).

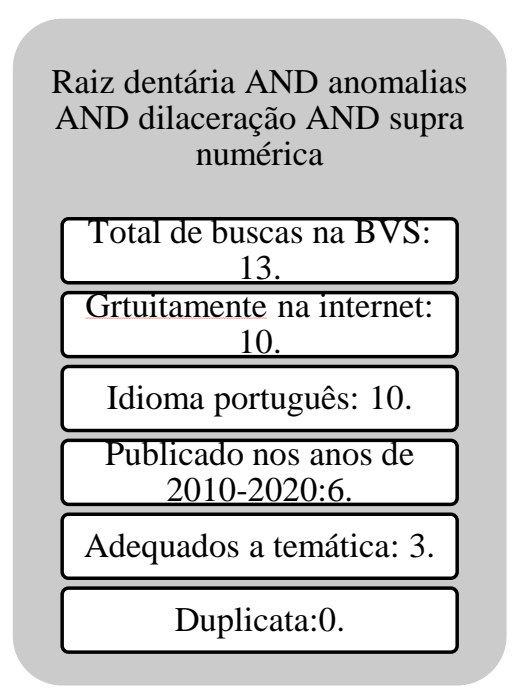

A Figura 2 expõe de forma sintetizada o processo de exclusão e classificação dos artigos que serviram de resultado dessa RIL. O esquema A referente à combinação de Raiz dentária AND anomalias AND assistência com um total de 79 artigos, decaindo conforme aplicados os termos de inclusão tendo como resultado final 1 artigos sem duplicação que se adeque a temática abordada.

Observou-se que a combinação B ao abordar a combinação Raiz dentária AND alterações AND tratamento foram forneceu um total de 25 artigos, sendo que após a aplicação dos filtros de busca foram selecionados 1 artigo adequado a temática e sem duplicação. No que se refere a combinação C Raiz dentária AND anomalias AND dilaceração AND supra numérica a princípio obteve-se um total de 13 artigos, após a filtragem apenas 3 se adequaram a temática da pesquisa.

A partir do dendograma de busca e seleção de artigos em bancos de dados representado pela Figura 2, foi possível formular o dendograma prisma, representado na Figura 3. Permitindo a realização de uma análise geral da quantidade de artigos disponibilizados na literatura acerca da presente temática. 
Figura 3: Dendograma prisma de busca de artigos em base de dados.
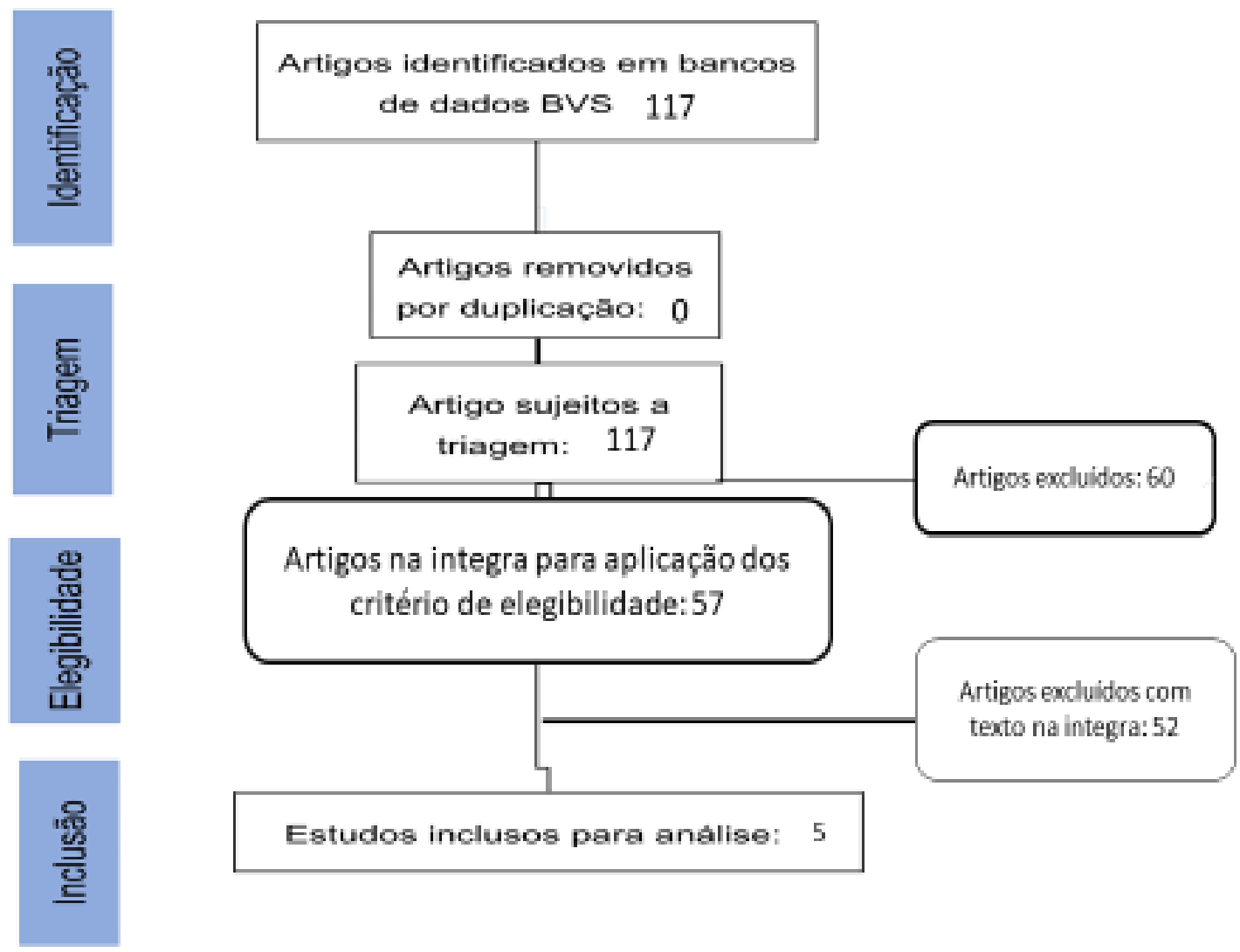

Fonte: Autores (2021).

Na Figura 3 foram expressados os resultados de busca de artigos em base de dados por meio do diagrama prisma, o qual expões os resultados globais da pesquisa, diante disso foi obtido antes das etapas de triagem um total de 117 artigos no processo de busca sem filtros na BVS

Ao passar pelo processo inicial de triagem sendo o primeiro filtro aplicado a disponibilização de artigos na íntegra como resultado elencou-se um valor de 57 artigos na integras sujeitados aos demais filtros sendo a disponibilidade de artigos em inglês e português nos bancos de dados uns dos filtros sequenciais, seguido pela disponibilidade de artigos publicados no período de 2010 a 2020 e pôr fim a adequação aos componentes temáticos de pesquisa, obtendo 5 artigos para a análise na revisão integrativa da literatura.

Observa-se com os resultados obtidos na pesquisa a carência de oferta de artigos relacionados Anomalias da raiz dilaceração e raiz supranumerária, haja vista que mesmo sem a aplicação dos filtros apenas 117 artigos foram elucidados com a pesquisa e a redução a um total de 5 artigos que correspondam aos critérios de inclusão, expressão a carência da temática, enaltecendo a necessidade de ampliar as pesquisas direcionadas a temática.

A partir dos resultados obtidos na segunda etapa da presente RIL, foi aplicado o formulário de Ursi (2005) pelo qual foi possível extrair os resultados dos artigos que ratificam sua fundamentação e validação científica para a elaboração e validação dos resultados expressos no presente estudo. Os resultados foram organizados conforme o Quadro 1. 
Quadro 1: Resultados da revisão integrativa em relação ao título do artigo, ano d epublicação, periódico, objetivos e conclusão.

\begin{tabular}{|c|c|c|c|}
\hline $\begin{array}{l}\text { Título e ano de } \\
\text { publicação }\end{array}$ & Periódico & objetivo & conclusão \\
\hline $\begin{array}{l}\text { A1 - Pre-operative } \\
\text { diagnostic } \\
\text { radiograph } \\
\text { interpretation by } \\
\text { general dental } \\
\text { practitioners for root } \\
\text { canal treatment. } \\
2012\end{array}$ & $\begin{array}{l}\text { Dentomaxillofacial } \\
\text { Radiology }\end{array}$ & $\begin{array}{l}\text { O objetivo deste estudo foi avaliar quais } \\
\text { características radiográficas radiculares os } \\
\text { dentistas gerais desejam interpretar, } \\
\text { determinar quais das características } \\
\text { radiográficas radiculares dentistas gerais } \\
\text { interpretam e quais eles perdem em uma } \\
\text { radiografia diagnóstica e correlacionam a } \\
\text { precisão com que os dentistas gerais são } \\
\text { capazes de interpretar características } \\
\text { radiográficas. }\end{array}$ & $\begin{array}{l}\text { Conclui-se deste estudo que os dentistas gerais são } \\
\text { capazes de } \\
\text { Detectar alterações radiográficas quando são } \\
\text { extensas, mas perdem o ligamento periodontal } \\
\text { mudanças de largura e lâmina dura. }\end{array}$ \\
\hline $\begin{array}{l}\text { A2 - Fused teeth in } \\
\text { the primary } \\
\text { dentition: clinical } \\
\text { case report. } \\
2020\end{array}$ & $\begin{array}{l}\text { Revista Gaúcha } \\
\text { Odontologia. }\end{array}$ & $\begin{array}{l}\text { O objetivo deste estudo é apresentar um } \\
\text { caso clínico de fusão de dois incisivos } \\
\text { inferiores decíduos, as perspectivas de } \\
\text { tratamento da anomalia e discutir sua } \\
\text { repercussão durante o desenvolvimento da } \\
\text { dentição. }\end{array}$ & $\begin{array}{l}\text { A oclusão da dentição decídua desempenha um } \\
\text { papel significativo na orientação da oclusão do } \\
\text { dentição permanente subsequente. O odontológico } \\
\text { mais adequado intervenção é o resultado de uma } \\
\text { abordagem multidisciplinar envolvendo } \\
\text { odontopediatria, radiologia, ortodontia e } \\
\text { odontologia restauradora, considerando o } \\
\text { indivíduo características de cada caso, bem como } \\
\text { as expectativas de cada paciente e seu grau de } \\
\text { cooperação com o dentista tratamento. }\end{array}$ \\
\hline $\begin{array}{l}\text { A3 - Dilaceração } \\
\text { radicular: relato de } \\
\text { caso clínico. } \\
2015\end{array}$ & $\begin{array}{l}\text { Revista brasileiras } \\
\text { de odontologia }\end{array}$ & $\begin{array}{l}\text { O objetivo deste trabalho é demostrar a } \\
\text { importância das imagens radiográficas e } \\
\text { tomográficas para o diagnóstico e } \\
\text { planejamento do tratamento da dilaceração } \\
\text { radicular por meio de um caso clínico. }\end{array}$ & $\begin{array}{l}\text { O exame tomográfico é de fundamental } \\
\text { importância para complementar o diagnóstico e } \\
\text { planejamento de tratamentos traumáticos e de } \\
\text { anomalias. No caso da dilaceração radicular, a } \\
\text { radiografia periapical, panorâmica e/ou oclusal são } \\
\text { eficientes para diagnosticar essa anomalia de } \\
\text { forma, porém como é um exame bidimensional de } \\
\text { uma estrutura tridimensional, é na tomografia } \\
\text { computadorizada que teremos maior precisão } \\
\text { quanto à angulação, o local em que ocorreu a } \\
\text { angulação e qual direção ela tomou, sendo essa a } \\
\text { melhor técnica radiográfica para obter o } \\
\text { planejamento e tratamento correto nesse caso e } \\
\text { com maior probabilidade de sucesso }\end{array}$ \\
\hline $\begin{array}{l}\text { A4 - Prevalence } \\
\text { assessment of root } \\
\text { dilaceration in } \\
\text { permanent incisors. } \\
2012\end{array}$ & $\begin{array}{l}\text { Dental Press Journal } \\
\text { of Orthodontics }\end{array}$ & $\begin{array}{l}\text { o objetivo do presente trabalho foi verificar } \\
\text { a prevalência da dilaceração radicular em } \\
\text { incisivos permanentes, em uma clínica de } \\
\text { radiologia odontológica da cidade de João } \\
\text { Pessoa/PB. }\end{array}$ & $\begin{array}{l}\text { De acordo com a metodologia utilizada e } \\
\text { resultados obtidos no presente trabalho, a dilatação } \\
\text { radicular dos incisivos, embora não muito comum, } \\
\text { é uma grande anomalia que afeta os dentes } \\
\text { anteriores, com uma prevalência de } 1,03 \% \text { da } \\
\text { amostra, os incisivos laterais são os mais } \\
\text { acometidos }(92,6 \%) \text {, principalmente nos incisivos } \\
\text { superiores }(78,0 \%) \text {, ocorrendo mais no terço apical } \\
(90,2 \%) \text { e direção distal da raiz }(95,1 \%) \text {. }\end{array}$ \\
\hline
\end{tabular}




\begin{tabular}{|l|l|l|l|}
\hline $\begin{array}{l}\text { A5 - Cemental tear: } \\
\text { A case report with } \\
\text { nonsurgical } \\
\text { periodontal therapy. }\end{array}$ & Revista Odonto & $\begin{array}{l}\text { Relatar um caso de dilaceração cementária, } \\
\text { uma condição periodontal rara caracterizada } \\
\text { pela separação total ou parcial do cemento } \\
\text { dental, abordando principalmente aspectos }\end{array}$ & $\begin{array}{l}\text { Em conclusão, o conhecimento da clínica e } \\
\text { características radiográficas da ruptura do cimento } \\
\text { samportantes em prática odontológica e terapia } \\
\text { periodontal não cirúrgica deve ser uma modalidade } \\
\text { de tratamento adequada e previsível para este lesão } \\
\text { incomum. }\end{array}$ \\
\hline
\end{tabular}

Fonte: Autores (2021).

O Quadro 1 expõe que 3 artigos selecionados foram publicados em 2012, um em 2015 e um em 2020, o que colabora com o fato da carência de artigos atualizados acerca da temática do presente estudo, haja vista que 4 de 5 artigos foram publicados há mais de 5 anos, expressando assim a necessidade de investir em publicações atualizadas sobre a temática e a importância desta RIL

Outro dado importante sobre o Quadro 1 são as exposições dos objetivos e conclusões dos artigos selecionados, já que estas partes dos artigos fizeram parte da produção do corpus textual que compõe o texto codificado a ser analisado pelo IRAMUTEQ gerando os resultados das classes discursivas a partir dos resultados do estudo, diante disso, elaborou-se o dendograma de Classificação hierárquica descendente que expões o processo de análise desenvolvida pelo IRAMUTEQ conforme Figura 4.

Figura 4: Dendograma de classificação hierárquica descendente (CHD).

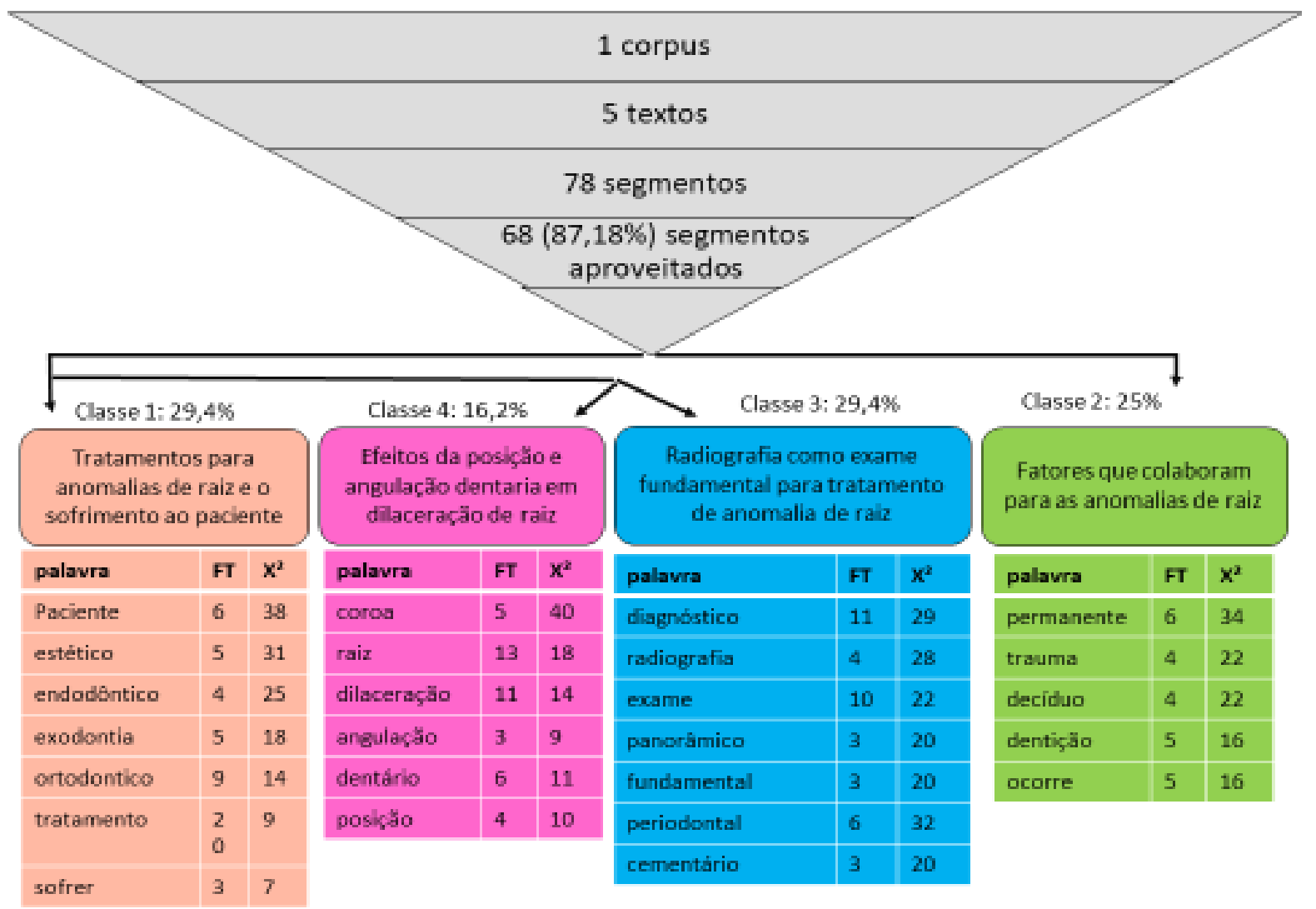

Fonte: Autores (2021). 
O IraMuTeQ preparou o Dendrograma através da CHD onde as palavras que obtiveram frequência igual ou maior a frequência média foram registradas e cada classe foi representada pelas palavras mais significativas e suas respectivas associações com a classe. Após a análise de identificação dos domínios textuais e interpretação dos significados, buscou-se nomear seus respectivos sentidos em classes descritas a seguir: 1) Tratamentos para anomalias de raiz e o sofrimento ao paciente; 2) Efeitos da posição e angulação dentária em dilaceração de raiz; 3) Radiografia como exame fundamental para tratamento de anomalia de raiz; 4) fatores que colaboram para as anomalias de raiz.

O corpus analisado no estudo é composto por 5 textos (UCI - unidade de contexto inicial) os quais se dividiram em 78 segmentos de texto que condizem com frases que apresentem nexo textual. Desses 78 segmentos 68 foram aproveitados para os resultados léxicos obtidos pela análise no Iramuteq tendo um total de $87,18 \%$ de aproveitamento o que é considerado um excelente aproveitamento. Para a criação de um dicionário de palavras, o programa emprega o teste do qui-quadrado ( $\mathrm{x}^{2}$ ), que revela a força associativa entre as palavras e suas respectivas classes. Essa força associativa é analisada quando o teste for maior que 3,84 representado por $\mathrm{p}$.

A partir do processo supracitado foram elencadas 4 classes derivadas das Unidades de Contexto Elementar (UCE) que resultou na criação de um dicionário reduzido com palavras homogêneas agrupadas segundo a ocorrência das palavras por meio de suas raízes etimológicas que colaboraram para dar sentido às classes discursivas expostas pelos 5 textos selecionados na RIL. No dendrograma exposto pela Figura 4, o corpus foi dividido em dois subcorpus. No primeiro subcorpus obteve-se a classe 1 individual que corresponde a 29,4\% do corpus com 7 UCE principais que fundamentam a base para a classe discursiva. O mesmo subcorpus apresentou outra subdivisão em classe 4 e 3, tais subdivisões, expressão a iteração das classes discursivas entre si e a proximidade léxica que elas apresentam. Do outro subcorpus obteve-se a classe 2 com 5 UCE, que correspondeu a $25 \%$ do total de UCE presentes no corpus.

\section{Tratamentos para anomalias de raiz e o sofrimento ao paciente}

O tratamento que pode ser proposto para reabilitar estética e funcionalmente estes pacientes é a combinação do tratamento ortodôntico e cirúrgico com o objetivo de melhor a qualidade de vida dos portadores desta doença. Trata-se de um tratamento longo, visto que tem extensos procedimentos ortodônticos, cirúrgicos e clínicos (Machado, C.,Pastor, I., Rocha, M., 2010). Uma proposta para o tratamento da má oclusão esquelética pode envolver cirurgia ortognática para corrigir a hipoplasia maxilar e extrair os dentes inclusos como é o caso dos dentes supranumerários. Deve-se também proceder à extração dos dentes decíduos de forma gradual e por regiões, expondo cirurgicamente os dentes permanentes para promover a sua erupção com o auxílio da tração ortodôntica quando necessário. (Serratine, 2007; Trindade, 2010; Machado, C.,Pastor, I., Rocha, M., 2010; Mehta, 2011; Kolokitha,O., Ioannidou, I., 2013).

Um diagnóstico precoce é fundamental para promover uma melhor qualidade de vida a estes pacientes por meio de uma reabilitação adequada, na qual o médico dentista tem um papel preponderante. Sendo o objetivo principal dos tratamentos dentários proporcionar uma aparência facial estética e uma oclusão funcional para o resto da vida (Chopra,R. et alii., 2012; Machado, C.,Pastor, I., Rocha, M., 2010).

O principal problema e sofrimento ao paciente, acontece durante a exodontia (extração dentária) em dente com raiz supranumerária. Os acidentes e complicações mais comuns na exodontia são: hemorragias; alveolites; dor; edema e trismo; injúria ao nervo alveolar inferior; infecções abrangendo espaços fasciais; injúrias em dentes adjacentes; fratura óssea da tuberosidade maxilar e/ou da mandíbula; comunicações bucossinusais; problemas periodontais em dentes adjacentes, e deslocamento de dentes para regiões anatômicas nobres. As manifestações pós-operatórias da exodontia dependem de uma série de fatores, como a técnica cirúrgica e a severidade da retenção, no entanto, a maioria dos pacientes relata sentir dor e, 
quando combinada com um processo inflamatório, os efeitos colaterais da cirurgia de terceiro molar podem ter um grande efeito sobre a qualidade de vida dos pacientes (Oliveira, 2006).

\section{Efeitos da posição e angulação dentária em dilaceração de raiz}

A dilaceração radicular ou curvatura anormal na raiz, impede a erupção dentária e é uma anomalia que pode ser causada por diferentes fatores etiológicos, dentre eles o traumatismo dentário na infância. O paciente portador de dilaceração radicular, com grau de dilaceração e inclinação coronária severos necessita de avaliação do grau de severidade da dilaceração radicular bem como da inclinação dentária feita através das medidas angulares do longo eixo dentário com a inclinação radicular (ângulo $\alpha$ ) e o raio (r), e do ângulo do longo eixo dentário com a linha APo (ângulo $\beta$ ). A incidência de dilaceração é maior nos incisivos centrais superiores (70,6\%), seguidos pelo incisivos laterais superiores (20.6\%) e incisivos laterais inferiores $(8,8 \%) 4$. A deformidade mais comum no incisivo central superior é a coroa angulada para cima e para vestibular (Torriani,2011).

A dilaceração radicular é um desvio abrupto do longo eixo da coroa ou da porção radicular do dente. Este desvio pode ocorrer em qualquer ponto ao longo do comprimento radicular - porção cervical, mediana ou porção apical, dependendo do estágio de formação radicular (Hamash, 2002). Pruett et al (1997), são usados dois parâmetros para definir a forma de curvatura dos canais: ângulo e raio da curvatura. $O$ raio da curvatura é o raio de uma circunferência que coincide com o caminho tomado pelo canal na área da curvatura mais abrupta. O ângulo é formado entre as linhas perpendiculares às tangentes que se interceptam no centro do círculo. $O$ raio de curvatura indica quão severa é a mudança de direção do canal, quando seu trajeto se desvia de uma linha reta.

Vale ressaltar que o tratamento e prognóstico do dente dilacerado dependem do grau de deformidade e formação da raiz do dente. Isso obriga o profissional a refletir sobre qual será a melhor conduta: se uma intervenção ortodôntica precoce, remoção da obstrução física se houver ou extração, ainda na dentição mista, com o objetivo de compor a harmonia do sorriso. As alternativas de tratamento de dentes permanentes dilacerados impactados incluem: exposição cirúrgica com tração ortodôntica, extração que pode ser seguida por (a) fechamento do espaço por mesialização do incisivo lateral com posterior restauração protética, (b) reposicionamento cirúrgico do incisivo central impactado, (c) autotransplante de um pré-molar à região e (d) restauração com implante ou ponte após cessação do crescimento (Costa, 2001).

\section{Radiografia como exame fundamental para tratamento de anomalia de raiz}

Para que um tratamento odontológico seja realizado com sucesso, é necessário solicitar uma série de exames que servirão para identificar, com maior precisão, a presença de doenças, lesões ósseas e até mesmo para o planejamento de conduta. Dentre esses exames, certamente, a radiografia odontológica é um dos mais pedidos. E isso porque as radiografias são instrumentos indispensáveis para vários casos, como os tratamentos de anomalias de raiz, supranumerária, ortodônticos, a realização de implantes e cirurgias na região do maxilar, além de auxiliarem na avaliação de patologias (doenças) e no processo de extração de um dente do siso. Assim sendo, a radiografia panorâmica, é a técnica radiográfica de escolha, por englobar o complexo maxilo-mandibular, fornecendo um meio extenso para visualizar e analisar os dentes e as estruturas de suporte em um único filme radiográfico (white,2017).

A radiografia panorâmica se caracteriza pela possibilidade da visão global de todos os elementos dentários da maxila e mandíbula, assim como de seus constituintes ósseos, além de ser, dentre os exames radiográficos, a mais aceitas por todas as crianças, segundo Araújo (1988). 
Na Radiografia Digital, os aparelhos de Raio-X são utilizados para o envio de informações para placas de circuito sensível, que recebem a radiação e mandam imagens direto para o computador. Como as placas são mais sensíveis do que os filmes radiográficos (utilizados nos exames tradicionais de Raio-X), o exame é realizado em muito menos tempo. Além disso, as imagens também são obtidas em muito menos tempo, o que torna o exame mais eficiente e seguro para os pacientes. Outro tipo são as radiografias periapicais utilizadas, de modo geral, para uma avaliação detalhada dos dentes de um paciente e permitem, por exemplo, uma análise cuidadosa de cáries. Por sua vez, as radiografias Interproximais são muito usadas para o diagnóstico de cáries entre os dentes (cárie interproximal), mesmo antes destas lesões se tornarem visíveis para o dentista sem o auxílio do Raio-X. As radiografias periapicais e as radiografias Interproximais são as mais comuns nas clínicas odontológicas do país (Pasler, 2011).

As radiografias panorâmicas, através de uma única imagem, produzem uma visão geral das arcadas dentárias dos pacientes, bem como, das regiões maxilar e mandibular. Elas são fundamentais para o planejamento inicial de um tratamento, tanto ortodôntico (para o alinhamento dental), quanto odontológico. É possível contar com radiografias panorâmicas digitais, parte importante dos avanços da ortodontia digital. Por fim, a radiografia oclusal é muito utilizada na odontopediatria, para o acompanhamento do crescimento dos dentes de uma criança, por exemplo. Além disso, ela pode ser útil para a análise de fraturas, dentes inclusos (que ficam abaixo da gengiva) e para analisar as raízes dos dentes (Neves, 2015). Os exames radiográficos quando bem indicados e realizados, juntamente com o exame clínico acurado, são fundamentais para o diagnóstico, sendo importantes para o planejamento e a terapêutica de cada caso (Carneiro, 2014).

\section{Fatores que colaboram para as anomalias de raiz}

A odontogênese compreende uma série de eventos celulares e moleculares altamente coordenados que culminam com a formação dos dentes. O processo requer interações recíprocas do epitélio oral com o ectomesênquima, cujo funcionamento envolve contatos intercelulares, interações mediadas por componentes da matriz extracelular e induções célula-a-célula por meio de fatores indutores. Diversas moléculas são responsáveis pela regulação do processo, dentre as quais merecem destaque os fatores de transcrição, fatores de crescimento e componentes da matriz extracelular. A influência de fatores genéticos e ambientais na etiologia das más oclusões representa tema de grande importância na odontologia. Quanto maior a contribuição genética na origem de uma irregularidade dentofacial, menor a possibilidade de preveni-la e, como regra, pior o prognóstico de tratamento ortodôntico/ortopédico. E os novos rumos da pesquisa em Odontologia caminham para o conhecimento do genótipo humano (Shalish, 2012).

Diversos estudos sugeriram uma tendência genética e hereditária na etiologia das anomalias dentárias de número, tamanho, posição, assim como nos distúrbios de erupção. Tais evidências provêm de investigações em famílias, em gêmeos monozigóticos, e da observação de associações na ocorrência de determinadas anomalias (Garib, 2015). Quando uma determinada irregularidade mostra uma prevalência aumentada em famílias de pacientes afetados, comparado às prevalências esperadas para a população em geral, credita-se à genética uma influência predominante na etiologia do problema.

Certas anomalias de forma, como dilacerações, raiz acessória, dente invaginado e taurodontismo, não causam alterações significativas à saúde bucal do paciente. Por outro lado, outras anomalias de forma, número, tamanho ou estrutura, em geral, exigem avaliação e, caso necessário, a intervenção por um profissional devidamente qualificado (FREITAS, et al., 2012).

\section{Conclusão}

Conclui-se, a partir deste trabalho, que as prevalências de anomalias dentárias nos estudos em revisão com pacientes concordam, via de regra, com a literatura atual, mesmo a literatura sendo de baixa publicação e produção. Caracterizada por 
complexos e precisos processos biológicos de substituição de dentes decíduos por dentes permanentes, a dentadura mista representa uma das manifestações de perfeição da natureza. Mas, como todo curso natural, o desenvolvimento da dentição pode mostrar algumas imperfeições e, no transcorrer da dentadura mista - com certa frequência -, o profissional depara-se com irregularidades odontogênicas: as anomalias dentárias. As anomalias expressam-se com distintos graus de severidade.

Da manifestação mais branda para a mais severa - representadas, respectivamente, desde o atraso cronológico na odontogênese até a ausência completa do germe dentário ou agenesia -, existe uma miríade de expressões, compreendendo as microdontias, os desvios na morfologia dentária e as ectopias.

A maior prevalência encontrada foi de dilaceração radicular $(38,1 \%)$, que se trata de uma anomalia com baixo poder de dano ao paciente, salvo necessidade de exodontia, situação na qual é mandatória a realização de exame radiográfico e, neste caso, seria diagnosticada a dilaceração e construído um plano cirúrgico específico e adequado. Deve-se, sim, fazer um bom exame inicial e acompanhamento do paciente e, a partir disso, observar achados clínicos que possam ser compatíveis com a presença de alguma anomalia dentária, para, então, lançar-se mão de um exame radiográfico para confirmação de diagnóstico.

Sendo um segundo passo no diagnóstico de anomalias dentárias, o exame radiográfico tem grande valor na construção de planos de tratamento adequados e específicos para a população pediátrica, possibilitando que se atue cedo sobre anomalias e condições danosas, procurando evitar seus possíveis desdobramentos negativos. Além disso, deve-se ressaltar que ainda existe uma carência de literatura específica sobre o presente tema de anomalias dentárias de raiz: dilaceração e raiz supranumerária. Novas técnicas vêm se desenvolvendo a cada dia e a atualização profissional torna-se indispensável para que se tenha uma boa decisão clínica, devolvendo assim a estética e função ou apenas preservando e acompanhando o caso.

\section{Referências}

Andrade, M., Weissman, R., Oliveira, M., et al. (2007). Tooth displacement and root dilaceration 10. after trauma to primary predecessor: an evaluation by computed tomography. Dent Traumatol; 23: 364-7.

Araújo, L. C. (1989). Radiografia Panorâmica e suas Aplicações em Odontopadiatria. São Paulo, 1989. 101p, Dissertação (mestrado). Faculdade de Odontologia de São Paulo, Universidade de São Paulo.

Azevedo C D B E, Ramos B. C., Pereira, J. L. C., Souza, P. S., Izar, B R S, \& Manzi, F. R. (2015). Dilaceração radicular: relato de caso clínico. Revista Brasileira de Odontologia;72.

Beyea, S. C., \& Nicoll, L. H. (1998). Writing in integrative review. AORN Journal, 67, 877-880

Botero, G. E.; Guzmán, H. A. M.; Méndez, G. A.; Pino, L. C.; Giraldo, J. E. R.; \& Botero, M. L. M. (2009). Estudio retrospectivo de anomalias dentales y alteraciones óseas de maxilares en niños de cinco a catorce años de las clínicas de la Facultad de Odontología de la Universidad de Antioquia. Revista Facultad de Odontología Universidad de Antioquia, Antioquia, 21(1), 50-64, 2009.

Carneiro, G. V. (2014). Estudo radiográfico da prevalência de anomalias dentárias por meio de radiografias panorâmicas em diferentes faixas etárias, Campo Grande. 76f. Tese (doutorado) - Programa de Pós-graduação em Saúde e Desenvolvimento na Região Centro-oeste.

Castro, L. et al. (2002). Estudo transversal da evolução da dentição decídua: forma dos arcos, sobressaliência e sobremordida, Revista Científica de Pesquisa Odontológica Brasileira, 16 (4), 367-373.

Cral, W. G. (2016). Achados incidentais em radiografias panorâmicas de Pacientes pré e pós-tratamento ortodôntico, 119 f. Dissertação (Mestrado em Ciências no Programa de Ciências Odontológicas Aplicadas) - Faculdade de Odontologia de Bauru, Universidade de São Paulo, São Paulo.

Chopra, R. et al., (2012). Hypodontia and Delayed Dentition as the Primary Manifestation of Cleidocranial Dysplasia Presenting with a Diagnostic Dilemma, Case Reports in Dentistry, pp.1-4.

Christophersen, P., Freund, M, \& Harild, L. (2005). Avulsion of primary teeth and sequelae on the 11. permanent successors. Dent Traumatol, 21, 320-3.

Costa, C. S. et al. (2001). Dilaceração radicular: tratamento cirúrgico e reabilitação estéticofuncional do paciente. BCI, 8(29), 76-80.

Dutra, S. R., Cabral, K., Lages Bem, et al. (2007). Dentes com dilaceração radicular: revisão de literatura e apresentação de caso clínico. Ortodontia SPO, 40(3), 216-21.

Faria, P. (2003). Prevalência das anomalias dentárias observadas em crianças de 5 a 12 anos de idade no município de Belém - um estudo radiográfico. Dissertação de Mestrado. São Paulo: Faculdade de Odontologia. 
Fernandes, L. M. (2000). Úlcera de pressão em pacientes críticos hospitalizados: uma revisão integrativa da literatura. Dissertação de mestrado, Universidade de São Paulo, Ribeirão Preto.

Freitas, D. Q.; Tsumurai, R. Y.; \& Machado Filho, D.N.S.P. (2012). Prevalence of dental anomalies of number, size, shape and structure. Revista Gaucha de Odontologia, Porto Alegre, 60(4), 437- 441, dez.

Garib, D. G., Zanella, N. L. M., \& Peck S. (2015). Associated dental anomalies: case report. J Appl Oral Sci.13(4), 431-6.

Hamasha, A. A.; Al-khateeb, T.; \& Darwazeh, A. (2002). Prevalence of dilaceration in Jordanian adults. IntEndod J, Oxford, 35(11), 910-912.

Jafarzadeh, H, \& Abbott, P. V. (2007). Dilaceration: review of an endodontic challenge. Jornal Endodontic 33(9), 1025-30.

Lucas, P. Y. X. (2019). Ocorrência de achados radiográficos em pacientes pediátricos / Phiscianny Yashmin Xavier Lucas. - Natal,.

Lo Biondo-Wood G, \& Haber J. (2001). Pesquisa em enfermagem: métodos, avaliação crítica e utilização. (4a ed.). Guanabara Koogan.

Miloglu, O, Cakici, F, Caglayan, F, et al. (2010). The prevalence of root dilacerations in a Turkish population. Med Oral Patol Oral Cir Bucal, 1 (15): 4414 .

Neves, I. R. (2015). LER: trabalho, exclusão, dor, sofrimento e relação de gênero. Um estudo com trabalhadoras atendidas num serviço público de saúde. Cad Saude Publica; 22(6), 1257-65.

Oliveira, L. B., Schmidt, D. B., Assis, A. F., Gabrielli, M. A. C., Hochuli-Vieira, E., \& Pereira Filho, V. A. (2006). Avaliação dos acidentes e complicações associadas à exodontia dos $3^{\circ}$ molares. Rev Cir Traumatol Buco-Maxilo-Fac. (2), 51-6.

Pasler, F. A.; \& Visser, H. (2011). Radiologia Odontológica, coleção atlas coloridos de odonto/ thierre. (2a ed,), p. $195-208$.

Paula, A. B. et al. (2008). / UNOPAR Cient., Ciênc. Biol. Saúde, Londrina, 10(1), 19-24, abr.

Polit, D. F., Beck, C. T., \& Hungler, B. P. (2004). Fundamentos de pesquisa em enfermagem: métodos, avaliação e utilização. (5a ed.). Artmed.

Silva, B. F., Costa, Led, Beltrão, R. V., et al. (2012). Prevalence assessment of root dilaceration in permanent incisors. Dental Press J Orthod 2012;17(6), 97-102.

Tommasi, M. H. M. Estomatologia Pediátrica. In: Tommasi, M. H. M. (2014). Diagnóstico em Patologia Bucal. (4. ed.). Elsevier, Cap. 29.

Torriani, D. D., Baldisseira, E , \& Goettem, M. L. (2011). Management of root dilaceration in acentral incisor after avulsion of primary tooth: a case report with a 6-year follow-up. Rev Odonto Cienc 2011; 26(4):355-8.

Trindade, A. et al . (2010). Displasia Cleidocraniana, Rev Bras Ciênc Saúde, 14(2), 73-76.

Theodorovicz, K. V.; Fernandes, A.; Westphalen, F. H.; \& Lima, A A S (2012).. Prevalência de raiz supranumerária em caninos de pacientes adultos jovens. Archives of Oral Research . 8(2).

Kolokitha, O., \& Ioannidou, I. (2013). A 13-year-old Caucasian boy with cleidocranial dysplasia: a case report, BMC Res Notes, 6(6), 1-6.

Machado,C. Pastor, I. \& Rocha, M. (2010).Características clínicas e radiográficas da displasia cleidocraniana-relato de caso, RFO, 15(3), 304-308.

Matos, P. C. (2015). Tipos de revisão de literatura. Faculdade de ciências agronômicas da UNESP. Botucatu, São Paulo.

Mehta, D. N., Vachhani, R. V., \& Patel, M. B. (2011). Cleidocranial dysplasia: a report of two cases, J Indian Soc Pedod Prev Dent, $29(3)$, pp. $251-254$.

Yaqoob, O, O’Neill, J, Gregg, T, et al. (2010). Management of uneruptedmaxillary incisors.

Prodanov, C.; \& Freitas, E. (2013). Metodologia do trabalho científico: métodos e técnicas da pesquisa e do trabalho acadêmico. (2. ed.). Feevale.

Santos, B. R. F. (2019). Aplicativo para mediar os cuidados básicos com recémnascidos no domicílio: Produção de tecnologia educacional baseado em evidencias. Monografia de conclusão de curso - Universidade do Estado do Pará, Belém, 2019.

Santos, M. A. R. C; \& Galvão, M. G. A. (2014). A elaboração da pergunta adequada de pesquisa. Residência pediátrica, 4 (2):53 - 56.

Serratine, A. \& Rocha, R. (2007). Displasia cleidocraniana-apresentação de um caso clínico, Arq Catar Med, 36 (1), $109-112$.

Souza, M. A. R.; Wall, M. L.; Thuler, A. C. M. C.; Lowen, I. M. V.; \& Peres, A. M. (2018). O uso do software Iramuteq na análise de dados em pesquisas qualitativas. Rev Esc Enferm USP, 52,. e03353, 2018.

Shalish, M., Peck, S, Wasserstein A, \& Peck L. (2012). Malposition of unerupted mandibular second premolar associated with agenesis of its antimere. Am J Orthod Dentofacial Orthop. 2012 Jan; 121(1), 53-6.

Torriani, D. D; Baldisseira, E. F. Z; \& Goettem, M. L. (2011). Management of root dilaceration in acentral incisor after avulsion of primary tooth: a case report with a 6-year follow-up. Rev Odonto Cienc 2011; 26(4):355-8.

White, S. C.; \& Pharoah J. M. (2017). Radiologia Oral. (5ª ed,). Elsevier, 2017. Cap. 18 